\title{
Analysis of Nanostructures with Scanning Transmission Electron Microscopy
}

\author{
Peter A. Crozier
}

Center for Solid State Science, Arizona State University, Tempe, AZ 87287-1704

Nanomaterials cover a diverse class of systems including high surface-area heterogeneous catalysts, quantum dots, nanoscale variations in interface and surface properties. Understanding the unique physical properties underlying nanomaterials and nanostructures requires the local structure and chemistry to be determined at the atomic scale. Scanning transmission electron microscopy (STEM) offers a powerful combination of techniques that may permit structural and compositional information to be obtained with better than $0.2 \mathrm{~nm}$ spatial resolution in the current generation of commercially available instruments. For STEM imaging, the probe is focused and scanned over the sample in a raster pattern and an image is formed by measuring a signal arising from the beam-specimen interactions. The elastically scattered fast electron signals can be employed to give bright or dark-field images. Alternatively, a spectroscopic signal can be measured and used to generate an elemental map. The probe can also be held stationary at one point in the object and an electron diffraction pattern or spectrum recorded. Under ideal conditions, composition and bonding information can be obtained from the electron energy-loss spectrum (EELS) with atomic column resolution.

High-angle annular dark-field imaging (so-called Z-contrast imaging) is a particularly powerful STEM technique for characterizing nanostructures [1]. The high-angle scattering is collected and used to form an image which has a strong dependence on atomic number. Moreover, because of the incoherent nature of the imaging process, atomic resolution Z-contrast images are generally simpler to interpret than an atomic resolution phase contrast image. The low-angle scattering can be measured simultaneously and used to form the energy-loss spectrum making the combined use of Z-contrast imaging and EELS a powerful approach for extracting structural and compositional information at the atomic level. Figure 1a shows an atomic resolution Z-contrast image of a $\mathrm{ZrB}_{2} / \mathrm{Si}$ interface recorded from a JEOL 2010F. The upper part of the image shows the Si dumb-bell units and the more intense lower section shows the $\mathrm{Zr}$ atom columns. The image clearly shows the registry between the atom positions, although the contrast from both the $\mathrm{Zr}$ and Si appears fuzzy at the interface.

Bright-field (BF) STEM imaging can also provide structural information that is in some ways complementary to the Z-contrast image. Figure $1 \mathrm{~b}$ and $1 \mathrm{c}$ are Z-contrast and BF STEM images (recorded at similar defocus) from another section of the $\mathrm{ZrB}_{2}$ interface. This pair of images can be used to compare the information in Z-contrast and BF STEM imaging. Clearly the Z-contrast image shows strong contrast for the heavy $\mathrm{Zr}$ atoms and very poor contrast for the lighter $\mathrm{Si}$ atoms. The BF image also shows the presence of amorphous material at the interface - this amorphous phase gives rise to diffuse contrast in the in the Z-contrast image. The BF signal shows much strong contrast from the crystalline regions of both materials reflecting the fact that coherent phase contrast microscopy is a powerful approach for picking out crystalline regions even when they are embedded in a thick amorphous matrix. 
The complementary image information is also apparent in the catalyst example of Figure 2 showing a Z-contrast and corresponding $\mathrm{BF}$ image recorded from a $\mathrm{Pd} / \mathrm{Al}_{2} \mathrm{O}_{3}$ catalyst. The Zcontrast technique is powerful for locating the position of the heavy-metal particles on the lightelement support. However, the atomic resolution images from such particles often provide very little structural information because the particles lie in random orientations away from strong channeling conditions. Phase contrast $\mathrm{BF}$ imaging shows lattice fringes over a much wider range of particle tilt. In this case, we see that the particle is crystalline, composed of several crystallite domains and has a well-defined orientation relationship with the $\mathrm{Al}_{2} \mathrm{O}_{3}$ substrate (see inset Fourier transform). The BF image can also provide information on surface structures on the nanoparticles. Figure $2 \mathrm{~b}$ shows the presence of an amorphous layer partially covering the $\mathrm{Pd}$ nanoparticle. EELS analysis revealed this to be a Si rich phase.

[1] S.J. Pennycook and P.D. Nellist, Impact of Electron and Scanning Probe Microscopy on Materials Research, (1999), 161-207, Kluwer Academic Publishers

[2] The author would like to thank Drs John Tolle and Rou-Jane Liu for the kind provision of samples

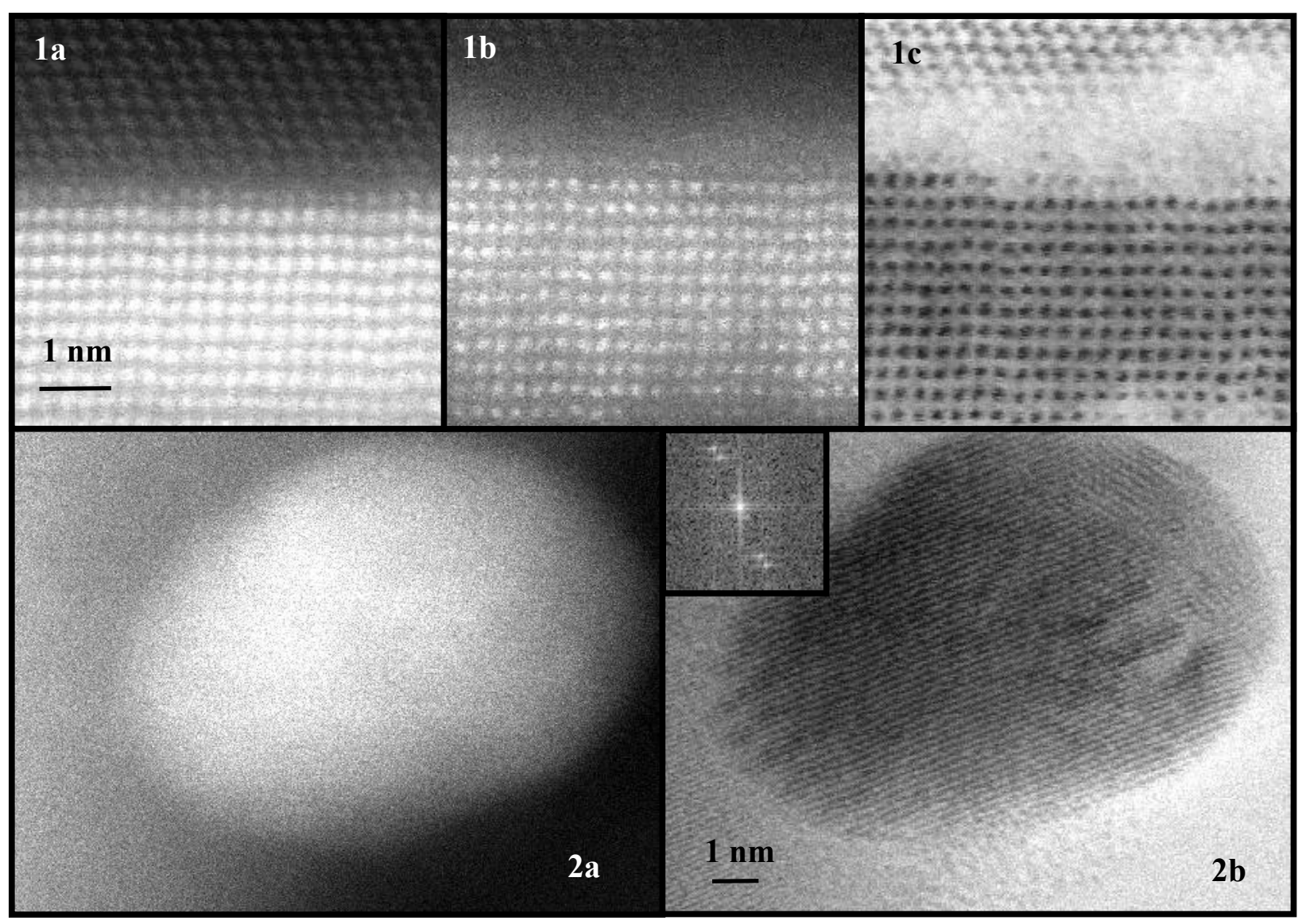

Figure 1: a) and b) Z-contrast image and c) bright-field STEM image from $\mathrm{ZrB}_{2} / \mathrm{Si}$ interface. Figure 2: a) Z-contrast image and b) $\mathrm{BF}$ STEM image from $\mathrm{Pd} / \mathrm{Al}_{2} \mathrm{O}_{3}$ nanoparticle. 